\title{
On the Convergence Behavior of Continued Fractions with Real Elements*
}

\author{
By Walter Gautschi
}

\begin{abstract}
We define the notion of transient (geometric) convergence rate for infinite series and continued fractions. For a class of continued fractions with real elements we prove a monotonicity property for such convergence rates which helps explain the effectiveness of certain continued fractions known to converge "only" sublinearly. This is illustrated in the case of Legendre's continued fraction for the incomplete gamma function.
\end{abstract}

1. Introduction. Continued fractions, as is well known, can be viewed in terms of infinite series. To describe the convergence behavior of a series it is useful to consider the notion of transient (geometric) convergence rate. Given a convergent series $\sum_{n=0}^{\infty} t_{n}$, the $n$th transient convergence rate is the quantity $\left|\rho_{n}\right|, n=1,2, \ldots$, where $t_{n}=\rho_{n} t_{n-1}$ (assuming $t_{n-1} \neq 0$ ). If $\lim _{n \rightarrow \infty}\left|\rho_{n}\right|=r, 0 \leqslant r \leqslant 1$, convergence is linear (geometric) with convergence rate $r$, if $0<r<1$, superlinear, if $r=0$, and sublinear if $r=1$. It is important to note, however, that these concepts are asymptotic in nature, hence not necessarily relevant for numerical (finite!) computation. Thus, a series need not be dismissed as useless, simply because it converges only sublinearly. The approach of $\left|\rho_{n}\right|$ to the limit 1 indeed may be so slow that the series has "converged to machine precision" long before $\left|\rho_{n}\right|$ reaches the neighborhood of 1. For this reason, convergence of a series ought to be judged on the basis of the complete sequence $\left\{\rho_{n}\right\}$ of convergence rates, and not just on the basis of asymptotic properties of $\rho_{n}$. In this connection, properties of monotone behavior significantly add to the understanding of the quality of convergence.

The purpose of this note is to prove a criterion for the sequence $\left\{\left|\rho_{n}\right|\right\}$ to be (ultimately) monotonically increasing, in the case where the partial sums of the series are convergents of a continued fraction with real elements. We illustrate the result with Legendre's continued fraction for the incomplete gamma function, which, though sublinearly convergent, provides an effective tool of numerical computation.

2. Continued Fractions and Infinite Series. We consider continued fractions of the form

$$
c=\frac{1}{1+} \frac{a_{1}}{1+} \frac{a_{2}}{1+} \cdots,
$$

where, for some integer $k_{0} \geqslant 1$,

$$
\begin{gathered}
\quad a_{k}>0 \text { for } 1 \leqslant k \leqslant k_{0}-1, \\
a_{k}<0 \text { and }\left|a_{k}\right| \leqslant \frac{1}{4} \quad \text { for } k \geqslant k_{0} .
\end{gathered}
$$

Received February 16, 1982.

1980 Mathematics Subject Classification. Primary 40A15; Secondary 33A70.

Key words and phrases. Convergence of real continued fractions, Legendre's continued fraction for the incomplete gamma function.

${ }^{*}$ Sponsored in part by the National Science Foundation under Grant MCS-7927158. 
It can be seen from Worpitzky's theorem (Henrici [3, p. 506]) that the tail of the continued fraction (2.1) beginning with the element $a_{k_{0}}$, hence also the complete continued fraction, converges. The infinite series

$$
s=\sum_{k=0}^{\infty} t_{k} \quad\left(t_{0}=1\right)
$$

is equivalent to the continued fraction (2.1) if its $n$th partial sum

$$
s_{n}=1+\sum_{k=1}^{n-1} t_{k}
$$

is equal to the $n$th convergent of $c$, for each $n=1,2,3, \ldots$. According to Euler,

$$
s_{1}=1, \quad s_{k+1}=s_{k}+t_{k}, \quad k=1,2,3, \ldots,
$$

where

$$
\left.\begin{array}{l}
\rho_{0}=0, \quad t_{0}=1, \\
\rho_{k}=\frac{-a_{k}\left(1+\rho_{k-1}\right)}{1+a_{k}\left(1+\rho_{k-1}\right)} \\
t_{k}=\rho_{k} t_{k-1}
\end{array}\right\} \quad k=1,2,3, \ldots .
$$

This represents a convenient algorithm for evaluating the continued fraction $c$, and is also useful for analyzing qualitative properties of convergence. Note indeed that the quantities $\rho_{n}$ in (2.6) yield the transient convergence rates $\left|\rho_{n}\right|$ of the series (2.3).

Slightly more convenient for analytical purposes are the quantities $\sigma_{k}=1+\rho_{k}$, which satisfy

$$
\sigma_{0}=1, \quad \sigma_{k}=\frac{1}{1+a_{k} \sigma_{k-1}}, \quad k=1,2,3, \ldots
$$

3. Convergence Behavior. Some first insights into the convergence behavior of the continued fraction (2.1) can be gained from the following lemma.

LEMMA 3.1. If the partial numerators $a_{k}$ in (2.1) satisfy (2.2), then the quantities $\sigma_{k}$ in (2.7) satisfy

$$
0<\sigma_{k}<1 \text { for } 1 \leqslant k \leqslant k_{0}-1
$$

and

$$
1<\sigma_{k} \leqslant \frac{2\left(k-k_{0}+2\right)}{k-k_{0}+3} \text { for } k \geqslant k_{0} .
$$

Proof. The inequalities (3.1) follow immediately from the positivity of $a_{k}$ and (2.7). To prove (3.2), we use induction. Since $-\frac{1}{4} \leqslant a_{k_{0}}<0$ and $0<\sigma_{k_{0}-1} \leqslant 1$, we have $1<\sigma_{k_{0}} \leqslant 4 / 3$, so that (3.2) is true for $k=k_{0}$. Assuming its truth for some $k \geqslant k_{0}$, we obtain

$$
1<\sigma_{k+1}=\frac{1}{1+a_{k+1} \sigma_{k}} \leqslant \frac{1}{1-\frac{1}{4} \frac{2\left(k-k_{0}+2\right)}{k-k_{0}+3}}=\frac{2\left(k-k_{0}+3\right)}{k-k_{0}+4}
$$

which is (3.2) with $k$ replaced by $k+1$. 
Lemma 3.1, in particular, implies $0<\sigma_{k}<2$, hence $-1<\rho_{k}<1$, for all $k \geqslant 1$. The series (2.3), therefore, has terms that are strictly decreasing in absolute value. Furthermore, by (3.1) and (3.2),

$$
-1<\rho_{k}<0 \text { for } 1 \leqslant k \leqslant k_{0}-1 \text {, and } 0<\rho_{k}<1 \text { for } k \geqslant k_{0},
$$

so that the series initially (if $k_{0}>1$ ) behaves like an alternating series and subsequently turns into a monotone series.

A more detailed description of convergence is provided by the following theorem.

THEOREM 3.1. If the partial numerators $a_{k}$ in (2.1) satisfy (2.2), and in addition $-\frac{1}{4} \leqslant a_{k+1} \leqslant a_{k}<0$ for $k \geqslant k_{0}$, then

(3.4) $-1<\rho_{k}<0$ for $1 \leqslant k \leqslant k_{0}-1$ and $\rho_{k+1}>\rho_{k}>0$ for $k \geqslant k_{0}$.

In particular,

$$
\lim _{k \rightarrow \infty} \rho_{k}=\rho, \quad \rho=\frac{1-\sqrt{1+4 a}}{1+\sqrt{1+4 a}},
$$

where $a=\lim _{k \rightarrow \infty} a_{k}$; the continued fraction (2.1) converges linearly, with convergence rate $\rho$, if $a>-\frac{1}{4}$, and sublinearly if $a=-\frac{1}{4}$.

Proof. The first inequalities in (3.4) have already been noted in (3.3). The others are equivalent to $\sigma_{k+1}>\sigma_{k}>1$ for $k \geqslant k_{0}$. Since $\sigma_{k}>1$, by (3.2), it suffices to prove

$$
\sigma_{k+1}>\sigma_{k} \text { for } k \geqslant k_{0} \text {. }
$$

We first show

$$
\sigma_{k-1}<\frac{2}{1+\sqrt{1-4\left|a_{k}\right|}} \text { for } k \geqslant k_{0} .
$$

This is true for $k=k_{0}$, since by (3.1) (and (2.7), if $k_{0}=1$ ) $\sigma_{k_{0}-1} \leqslant 1$, while the expression on the right of (3.7) is greater than 1. Using induction, assume that (3.7) holds for some $k \geqslant k_{0}$. Then

$$
\begin{aligned}
\sigma_{k} & =\frac{1}{1+a_{k} \sigma_{k-1}}<\frac{1}{1-\left|a_{k}\right| \frac{2}{1+\sqrt{1-4\left|a_{k}\right|}}} \\
& \leqslant \frac{1}{1-\frac{2\left|a_{k+1}\right|}{1+\sqrt{1-4\left|a_{k+1}\right|}}},
\end{aligned}
$$

where in the last inequality we have used $\left|a_{k+1}\right| \geqslant\left|a_{k}\right|$. Now observe that, for any $\alpha \leqslant \frac{1}{4}$,

$$
\begin{aligned}
\frac{1}{1-\frac{2 \alpha}{1+\sqrt{1-4 \alpha}}} & =\frac{1+\sqrt{1-4 \alpha}}{1+\sqrt{1-4 \alpha}-2 \alpha} \\
& =\frac{1-(1-4 \alpha)}{1-(1-4 \alpha)-2 \alpha(1-\sqrt{1-4 \alpha})}=\frac{2}{1+\sqrt{1-4 \alpha}}
\end{aligned}
$$


Using this in (3.8), with $\alpha=\left|a_{k+1}\right|$, yields (3.7) with $k$ replaced by $k+1$, and thus establishes (3.7) for all $k \geqslant k_{0}$.

Now (3.6), in view of (2.7), is equivalent to

$$
\frac{1}{1+a_{k+1} \sigma_{k}}>\sigma_{k} \text { for } k \geqslant k_{0}
$$

which in turn, since $1+a_{k+1} \sigma_{k}>0$ and $a_{k+1}<0$ for $k \geqslant k_{0}$, is equivalent to

$$
\left|a_{k+1}\right| \sigma_{k}^{2}-\sigma_{k}+1>0 \text {. }
$$

The quadratic function $\left|a_{k+1}\right| t^{2}-t+1$ is convex and has two real zeros $t_{1, k+1}<$ $t_{2, k+1}$, the smaller of which is

$$
t_{1, k+1}=\frac{2}{1+\sqrt{1-4\left|a_{k+1}\right|}} .
$$

By (3.7), $\sigma_{k}<t_{1, k+1}$, hence $\left|a_{k+1}\right| \sigma_{k}^{2}-\sigma_{k}+1>0$, which implies $\sigma_{k+1}>\sigma_{k}$. This proves (3.6).

Since the sequence $\left\{a_{k}\right\}$ is monotonically decreasing for $k \geqslant k_{0}$, and bounded below by $-\frac{1}{4}$, the $\operatorname{limit} \lim _{k \rightarrow \infty} a_{k}=a$ exists, and $-\frac{1}{4} \leqslant a<0$, since $a_{k_{0}}<0$. Similarly, $\lim _{k \rightarrow \infty} \rho_{k}=\rho, 0<\rho \leqslant 1$, and $\lim _{k \rightarrow \infty} \sigma_{k}=\sigma$ with $\sigma=1+\rho$. Going to the limit $k \rightarrow \infty$ in (2.7) then gives

$$
\sigma=\frac{1}{1+a \sigma}, \quad \sigma=\frac{2}{1 \pm \sqrt{1+4 a}} .
$$

Since $\sigma \leqslant 2$ and $-\frac{1}{4} \leqslant a<0$, the minus sign in the last equation for $\sigma$ cannot hold (unless $a=-\frac{1}{4}$ ), and we conclude that

$$
\sigma=\frac{2}{1+\sqrt{1+4 a}}, \quad \rho=\sigma-1=\frac{1-\sqrt{1+4 a}}{1+\sqrt{1+4 a}},
$$

which is (3.5). The last statement of the theorem is an immediate consequence of (3.5). This completes the proof of Theorem 3.1.

4. Truncation. In practice, the continued fraction (2.1) is evaluated by carrying out (2.5) and (2.6) for $k=1,2, \ldots, n$ and taking $s_{n+1}$ to approximate the value of $s$ (or $c$ ) of the continued fraction. It is important, then, to be able to choose $n$ in such a way that $s_{n+1}$ approximates $s$ to any prescribed accuracy.

Assuming first $k_{0}=1$, hence $0<\rho_{k}<1$ by (3.3) and $0<t_{k}<1$, it follows from a result of Merkes [4, Eq. (12)] that

$$
\left|s-s_{n+1}\right| \leqslant \frac{1+\rho_{n}}{1-\rho_{n}} t_{n} .
$$

This suggests the following stopping rule: Given a prescribed (relative) accuracy $\varepsilon$, stop the recursion (2.6) at the first integer $k=n$ for which

$$
\left(1+\rho_{n}\right) t_{n} \leqslant\left(1-\rho_{n}\right) s_{n+1} \varepsilon .
$$

By (4.1), this implies $\left|s-s_{n+1}\right| \leqslant s_{n+1} \varepsilon$, hence

$$
\frac{\left|s-s_{n+1}\right|}{s+\left|s_{n+1}-s\right|} \leqslant \frac{\left|s-s_{n+1}\right|}{s_{n+1}} \leqslant \varepsilon,
$$


from which $\left|s-s_{n+1}\right| \leqslant s \varepsilon+\left|s_{n+1}-s\right| \varepsilon$, that is,

$$
\left|\frac{s-s_{n+1}}{s}\right| \leqslant \frac{\varepsilon}{1-\varepsilon} .
$$

Our stopping rule therefore achieves the desired accuracy, at least asymptotically for $\varepsilon \rightarrow 0$.

To avail oneself of this simple stopping rule, when $k_{0}>1$, one ought to first evaluate the "tail"

$$
c_{k_{0}}=\frac{1}{1+} \frac{a_{k_{0}}}{1+} \frac{a_{k_{0}+1}}{1+} \cdots
$$

of the continued fraction (2.1), to which Merkes' result applies, and then compute

$$
c_{k}=\frac{1}{1+a_{k} c_{k+1}} \quad \text { for } k=k_{0}-1, k_{0}-2, \ldots, 1,
$$

to get the complete continued fraction $c=c_{1}$. Since $c_{k_{0}}>0$ and $a_{k}>0$ for $k<k_{0}$, the computation in (4.5) involves the addition of positive numbers and division, hence only numerically stable operations.

5. An Example. Theorem 3.1 is applicable to Legendre's continued fraction for the incomplete gamma function,

$$
\begin{gathered}
(x-\alpha+1) x^{-\alpha} e^{x} \Gamma(\alpha, x)=\frac{1}{1+} \frac{a_{1}}{1+} \frac{a_{2}}{1+} \cdots, \\
a_{k}=\frac{k(\alpha-k)}{(x-\alpha+2 k-1)(x-\alpha+2 k+1)}, \quad k=1,2,3, \ldots,
\end{gathered}
$$

which is used in [1, p. 475], [2] to compute the incomplete gamma function in the domain $D: x \geqslant 1.5,-\infty<\alpha \leqslant x+\frac{1}{4}$. Assuming $\alpha$ not a positive integer (otherwise, the continued fraction (5.1) would terminate and our assumption (2.2) would be violated), we have for $(x, \alpha) \in D$

$$
k_{0}= \begin{cases}1 & \text { if } \alpha<1, \\ 1+[\alpha] & \text { if } \alpha>1 .\end{cases}
$$

If $k \geqslant k_{0}$, the condition $\left|a_{k}\right| \leqslant \frac{1}{4}$ is equivalent to $(x-\alpha)^{2}+4 k x \geqslant 1$, hence satisfied if $x \geqslant \frac{1}{4}$ (since $k \geqslant 1$ ). An elementary calculation furthermore shows that $\left|a_{k+1}\right| \geqslant\left|a_{k}\right|$ for $k \geqslant k_{0}$ whenever $x \geqslant \frac{1}{2}$. It follows, in particular, that all assumptions of Theorem 3.1 are satisfied when $(x, \alpha) \in D$. Since clearly $a=\lim _{k \rightarrow \infty} a_{k}=$ $-\frac{1}{4}$, we are in a case of sublinear convergence. (This is also noted by Henrici [3, p. 629] by way of a different analysis.) Nevertheless, the continued fraction is known to be quite useful as a computational tool, at least in a domain such as $D$. The reason for this is readily understood on the basis of Theorem 3.1: Although the transient convergence rates $\rho_{k}$ eventually increase monotonically to 1 , the limit is approached quite slowly. We can see this from Table 5.1 which, in the case of the continued fraction (4.4), and for selected $x$ and $\alpha$, displays the values of

$$
n_{\nu}=\max \left(k:\left|\rho_{k}\right| \leqslant \frac{\nu}{4}\right) \quad \text { and } \quad \varepsilon_{\nu}=\frac{4+\nu}{4-\nu} t_{n_{\nu}}, \quad \nu=1,2,3 \text {. }
$$


TABLE 5.1

Convergence behavior of the continued fraction (5.1)

( Numbers in parentheses indicate decimal exponents.)

\begin{tabular}{rlrlrcrc}
\hline$x$ & \multicolumn{1}{c}{$\alpha$} & $n_{1}$ & $\varepsilon_{1}$ & $n_{2}$ & $\varepsilon_{2}$ & $n_{3}$ & $\varepsilon_{3}$ \\
\hline 1.5 & 1.75 & 3 & $1.9(-3)$ & 13 & $2.9(-7)$ & 75 & $6.7(-18)$ \\
& .875 & 3 & $9.2(-4)$ & 13 & $1.2(-7)$ & 75 & $2.7(-18)$ \\
& 0.0 & 3 & $6.8(-3)$ & 13 & $1.4(-6)$ & 75 & $3.7(-17)$ \\
& -3.5 & 3 & $7.3(-3)$ & 13 & $1.4(-6)$ & 75 & $3.5(-17)$ \\
& -7.0 & 4 & $8.9(-4)$ & 15 & $5.8(-8)$ & 79 & $5.5(-19)$ \\
5.0 & 5.25 & 9 & $1.9(-8)$ & 41 & $5.4(-21)$ & 243 & $2.9(-56)$ \\
& 2.625 & 10 & $1.0(-9)$ & 41 & $7.3(-22)$ & 243 & $3.0(-57)$ \\
& 0.0 & 10 & $9.8(-10)$ & 42 & $2.9(-22)$ & 244 & $1.6(-57)$ \\
& -10.5 & 9 & $1.9(-8)$ & 42 & $1.6(-21)$ & 244 & $8.5(-57)$ \\
& -21.0 & 12 & $2.7(-11)$ & 48 & $1.2(-25)$ & 254 & $8.8(-62)$ \\
10.0 & 10.25 & 18 & $1.1(-16)$ & 81 & $1.2(-41)$ & 483 & $3.0(-112)$ \\
& 5.125 & 18 & $8.6(-17)$ & 81 & $7.1(-42)$ & 483 & $1.5(-112)$ \\
& 0.0 & 20 & $8.0(-20)$ & 83 & $8.1(-45)$ & 485 & $1.9(-115)$ \\
& -20.5 & 19 & $1.7(-17)$ & 83 & $6.9(-43)$ & 485 & $1.6(-113)$ \\
& -41.0 & 24 & $1.5(-22)$ & 95 & $5.0(-51)$ & 504 & $4.2(-123)$ \\
20.0 & 20.25 & 36 & $3.0(-33)$ & 161 & $4.8(-83)$ & 963 & $2.9(-224)$ \\
& 10.125 & 36 & $1.2(-33)$ & 162 & $5.2(-84)$ & 964 & $3.1(-225)$ \\
& 0.0 & 40 & $2.9(-40)$ & 165 & $3.4(-90)$ & 967 & $1.6(-231)$ \\
& -40.5 & 38 & $4.3(-35)$ & 165 & $8.8(-86)$ & 968 & $3.4(-227)$ \\
& -81.0 & 48 & $3.1(-45)$ & 188 & $1.2(-101)$ & 1004 & $6.8(-246)$ \\
& & & & & & &
\end{tabular}

Note that by virtue of (4.1), and the fact that $1<s=c_{k_{0}} \leqslant 2$ (cf. [3, Theorem 12.3c]),

$$
\left|\frac{s-s_{n_{\nu}+1}}{s}\right| \leqslant\left|s-s_{n_{\nu}+1}\right| \leqslant \varepsilon_{\nu}, \quad \nu=1,2,3 .
$$

Thus, for example, if $x=5, \alpha=0$, by the time the transient convergence rate has risen to $\frac{1}{2}$, the continued fraction has already converged to within a (relative) error of about $3 \times 10^{-22}$.

Department of Computer Sciences

Purdue University

West Lafayette, Indiana 47907

1. W. GaUtschi, "A computational procedure for incomplete gamma functions," ACM Trans. Math. Software, v. 5, 1979, pp. 466-481.

2. W. GautschI, "Algorithm 542-Incomplete gamma function," ACM Trans. Math. Software, v. 5, 1979, pp. 482-489.

3. P. Henrici, Applied and Computational Complex Analysis, Vol. 2, Wiley, New York, 1977.

4. E. P. Merkes, “On truncation errors for continued fraction computations," SIAM J. Numer. Anal., v. 3, 1966, pp. $486-496$. 